\title{
Minecraft Education Edition's Ability to Create an Effective and Engaging Learning Experience
}

\author{
Vennela Panja ${ }^{1}$ and Jill Berge ${ }^{1 \#}$ \\ Lake Washington High School, Kirkland, WA, USA \\ \#Advisor
}

\section{$\underline{\text { ABSTRACT }}$}

Effective game-based learning is possible and incredibly important, as it provides effective, engaging, studentcentered learning. While it has been successful for history learning, effective game-based learning for STEM subjects is scarce. This study explored Minecraft Education Edition's chemistry features and their ability to create an effective and engaging learning experience, as Minecraft's game mechanics along with Education Edition's chemistry features may provide a solution to the lack of effective game-based learning. The study was conducted with a lesson in Minecraft Education Edition, and participants took a quiz and reflection survey after the lesson to gauge Minecraft Education Edition's viability as a learning tool. While the data in the study was too limited to garner a conclusion on how Minecraft Education Edition affects the engagement and effectiveness of a lesson, it provides insight that not all students/players engage with Minecraft the same way and discusses many factors that should be considered when creating a Minecraft-based education model.

\section{Literature Review}

Throughout the past, many have implemented video games in education. This isn't surprising: game-based learning is a promising area. A peer-reviewed study reveals as early as 2006, teachers have been increasingly interested in implementing game-based learning, and this interest rises with the popularity of digital games (Burgos, Tattersall, and Koper, 2006). Additionally, over $65 \%$ of surveyed U.S. teachers in 2008 were interested in using games in the classroom (Watson, Mong, \& Harris, 2011). Many non-scientific articles report on the promise of games in education, arguing game-based learning should be implemented in classrooms as it is beneficial to students (Stemfuse, 2014; Gray, 2018). This is elaborated by multiple peer-reviewed sources, such as a paper by Arena (2015) and a study by Coleman and Money (2019), which reveal game-based learning can create student-centered learning experiences. Like video games, student-centered learning focuses on a participant's experience through engagement, providing an effective learning experience (Arena, 2015). This is reinforced by Coleman and Money (2019), who state studentcentered learning induces active learning and a deeper understanding of subject material. As they provide beneficial learning experiences, it is important to implement student-centered learning models in the classroom.

Furthermore, video games are designed to engage and provide a positive effect for players, and they can induce the psychological state of "flow", fully immersing a person with an activity, providing them maximum engagement, and completely driving them to their goal (Kiili, 2005). This is an optimal experience for learning, as Kiili's (2005) peer-reviewed study states flow is integral in creating a successful game-based learning experience and the future game-based learning promises involves engaging and motivating students throughout the game. Arena (2015) further connects flow to student-centered game-based learning, stating video games provide an environment where a student can grow with safe room for failure, providing feedback on a task and matching their learning abilities as they progress through the game, engaging the student further. If an instance of game-based learning had these elements, it would provide a fun and effective experience (Arena, 2015). 
Multiple case studies reveal game-based history learning has been successful. Watson, Mong, and Harris (2011) conducted a peer-reviewed case study on a high-school classroom utilizing the game Making History. During class periods where the game was played, students reported the class was more engaged with the lesson, with very few instances of nonengagement (Watson et al, 2011). Students also stated the game was a "pretty fun learning experience" (Watson et al., 2011). Additionally, "non-gamers" successfully played and learned through the game, which is important as in a classroom setting, not all students would be familiar with video games (Watson et al, 2011). In addition, social studies education professionals Maguth, List, and Wunderle (2015) reveal the game Age of Empires II, utilized in a similar way as Making History, was viable in the classroom, and games can be used for learning as long as they directly connect to the subject being taught. Age of Empires II allowed students to connect their experience with the game to the material taught in class (Maguth et al., 2015). This "brought world history, its issues, and people to life for students", greatly engaging them (Maguth et al., 2015). In addition, Shree, DeVane, and Durga's (2008) peer-reviewed study on designing beneficial game-based learning reveals Civilization III, an open-ended game, was able to create an incredibly positive learning experience during observation of several students playing it. Students were able to retain the information they were asked to recall and reported having incredible fun compared to prior school experience (Shree et al., 2008). From game-based learning's success with history and as these instances provided an effective and engaging learning experience, it is clear these games create a student-centered learning experience.

As described by Burgos, Tattersall, and Koper's (2006) peer-reviewed study on repurposing existing games for learning, good education-centric games offer a balance between interaction and education, meaning they offer student engagement which creates a sense of fun, while still effectively teaching material. However, many instances of game-based learning do not fulfill this balance and are not successful in education. According to Arena (2015), they tend to be closer to glorified schoolwork, with "fun" seemingly forced into the learning experience. This is described as a "chocolate-covered broccoli" experience, mismatching entertainment with education, but only appearing enjoyable, leaving students with an ineffective and unengaging learning experience (Arena 2015). Additionally, Kiili's (2005) peer-reviewed study on creating game-based learning models states many games for education employ a "drill and practice" principle. This forces students to engage in trial and error, rather than genuinely attempting to solve problems they are presented with (Kiili, 2005). As students do not use their knowledge to solve these puzzles, this does not provide an effective learning experience (Kiili, 2005). It is clear video games themselves are not the problem, as there have been many successful implementations of game-based learning. The issue likely resides in the games' designs. Video game design that includes an engaging and educational experience is essential to creating a successful implementation of game-based learning. While history game-based learning has been successful, its success was largely due to the narrative history provides by default, which carries over to the design of history-based games (Watson et al, 2011; Maguth et al, 2015). Contrasting history's success, viable gamebased learning experiences remain scarce for STEM (science, engineering, technology, and math), which are incredibly important to learn effectively as they are involved with numerous subjects and careers. STEM learning requires a different method of learning than history-based games, as STEM subjects do not provide an engaging narrative, so other mechanics must provide this engagement. Current STEM education games, such as the University of Colorado Boulder's (2021) PhET simulations, have potential as supplementary education tools, but invoke "drill and practice", creating an ineffective learning experience. This reveals a gap in research pertaining to game-based learning: STEM game-based learning remains lacking in engagement and effectiveness, and there is little information on how it can be successfully implemented.

While it may seem difficult to implement game-based learning for STEM, one game may provide a solution. Minecraft, created by game company Mojang, is an open-world, sandbox game that allows players to explore and develop a "world" to their liking (Hofer, 2015). It is also open-ended, similar to Civilization III, which was previously used successfully in history-based learning, indicating this mechanic would create a more beneficial learning experience (Shree et al., 2008) It is incredibly popular, with over 100 million players, indicating students may be familiar with it (Mojang, 2009; Hofer, 2015). In 2016, Mojang released Minecraft Education Edition, a version of 
Minecraft that was functionally the same, with the same game design mechanics, but with added features for teaching and learning. As Minecraft Education Edition's mechanics are the same as Minecraft's, the two titles will be used interchangeably in this paper. The majority of these features involved the STEM subject chemistry, adding elements of the periodic table, and in-game lab equipment to create elements, compounds, and chemical reactions (Mojang, 2016). Many studies have identified Minecraft Education Edition's features as a potentially beneficial tool in teaching chemistry. Chemistry is also highly popular as a subject to teach in Minecraft, likely because it includes chemistry features with no need to implement additional ones. (UWIRE, 2019). Additionally, according to Nebel, Schneider, and Rey's peer-reviewed study on Minecraft's past usage, Minecraft has been used in education, teaching many subjects including chemistry (Nebel, Schneider, \& Rey, 2015). In addition, Minecraft's game design mechanics give it advantages for game-based learning: its immersive visual digital environment provides players with engagement (Nebel et.al., 2015). Minecraft's immersion goes further: Sean C. Duncan's (2011) peer-reviewed study on Minecraft's potential shows it achieves flow between the anxiety from possible in-game death found in many games and Minecraft's lack of clear goals or narrative, providing a potential solution. This shows Minecraft's game mechanics may close the gap STEM games face, providing flow without a narrative. These elements have the potential to create a student-centered game-based learning experience.

Furthermore, Minecraft modules for teaching STEM subjects exist. Within Minecraft, users can create "maps", in which Minecraft's mechanics are used as a base, and additional mechanics and goals are integrated, building a game within a game. These "maps" provide fixed goals and narratives, which Minecraft does not provide aside from the objective of "survive and explore" (Duncan, 2011; UWIRE, 2019). According to an article by UWIRE text (2019), education-based maps are common. Unfortunately, these maps widen the gap good STEM games need to fill. Current user-created STEM-based Minecraft maps, like The Human Body by GoE-Craft (2020), which provides a lesson on biology and anatomy, and Astronaut Training Center by Shapescape (2020), meant to teach students about physics and astronomy, are ineffective, and mismatch education with Minecraft, creating a chocolate-covered broccoli situation. These maps tend to ignore Minecraft's game design mechanics in favor of the lesson's goals and don't use Minecraft to its fullest potential for education. Moreover, while there are numerous Minecraft-based lesson maps, most information on how to use Minecraft in the classroom comes from the same community that created the previously mentioned ineffective maps, and academic research on the subject is virtually nonexistent (Hofer, 2015). As Minecraft's design can create flow and immersion without a narrative, an implementation of Minecraft Education Edition utilizing Minecraft's base game design mechanics could prove to be very effective in teaching, making a more effective and engaging lesson.

To test Minecraft Education Edition's viability as a learning tool, the following research question was composed: How does the implementation of Minecraft Education Edition's game design mechanics affect a chemistry lesson's effectiveness and engagement?

\section{Methods}

LWSD, a school district in Washington state, provides Minecraft Education Edition to students for free. Additionally, Minecraft Education Edition's in-game lab equipment involves high school level chemistry concepts, so a high school in LWSD was chosen for the study.

Ideally, a single-classroom observation method would have been performed, similar to Maguth et al.'s (2011) and Watson et al.'s (2015) case studies. However, due to COVID-19 related restrictions, only students with a personal computer could download Minecraft Education Edition, and in-person observation was not possible. Therefore, a "Remote Observation" method was used, emulating the observation method used in these studies despite these limitations.

This method involved three parts:

1. Lesson in Minecraft Education Edition

2. Post-lesson Quiz 
3. Reflection survey

A link to the lesson download and copies of the post-lesson quiz and reflection survey can be found in the appendices.

\section{Researcher-created Lesson}

\section{Lesson Framework/Outline:}

Participants were provided a download of a Minecraft Education Edition map created by the researcher. Many aspects of Minecraft Education Edition and game-based learning were considered as the researcher created the lesson. Duncan's (2011) study states Minecraft's basic goals are "survive and explore", and like other education Minecraft maps, an additional goal was added in the lesson - to learn chemistry concepts. Minecraft Education Edition adds ingame chemistry lab equipment, and other chemistry items, such as physical representations of elements of the periodic table, and chemical compound items (Figure 1).

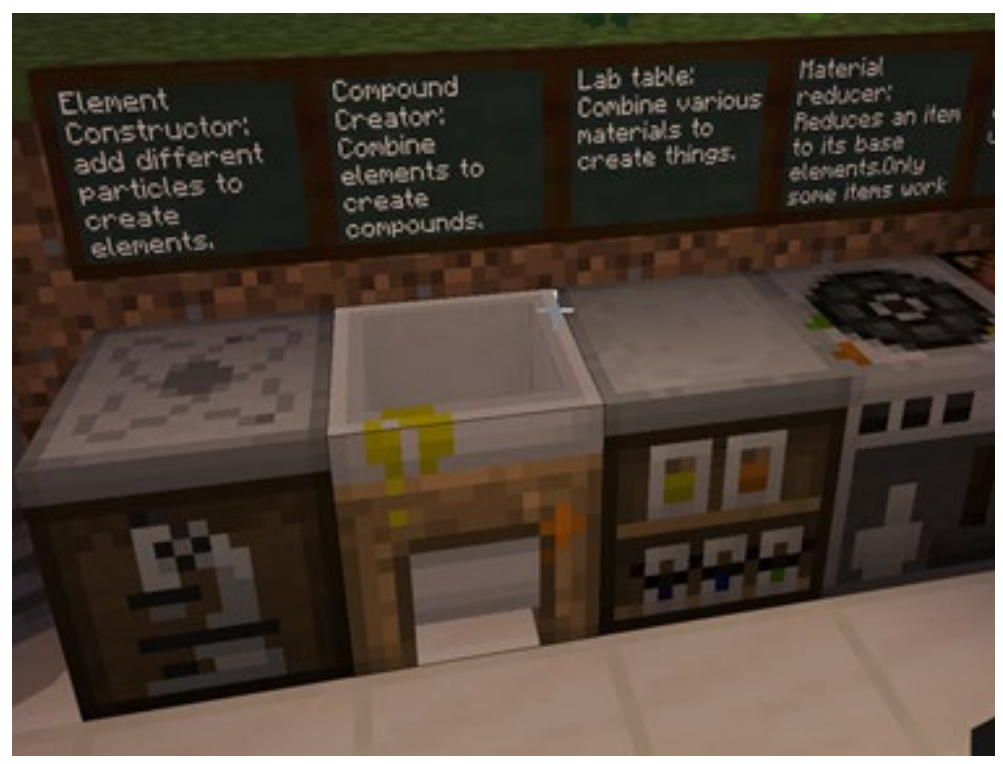

Figure 1: Minecraft Education Edition's lab equipment with descriptions.

Some uses of Minecraft Education Edition's items benefit the default Minecraft gameplay experience, as they can be used to create items that assist in survival and exploration. Burgos, Tattersall, and Koper's (2006) study on the repurposing of existing games for digital learning states game-based education must lead students to voluntarily interact with the puzzles or activities in the lesson. As the chemistry equipment in Minecraft Education Edition is useful to survival and exploration, if a goal of "learn chemistry subjects" was added to the default goals of "survive and explore", they could naturally mesh, avoiding a chocolate-covered broccoli situation (Duncan, 2011). Additionally, as flow is integral to game-based learning, it is essential to implement in a learning model for Minecraft Education Edition (Kiili, 2005). Kiili states activities relating to the main goal increase useability and flow, as additional activities and goals distract from the main task and decrease learning potential, so different stations that corresponded to the lesson's goal of learning chemistry were implemented as the lesson's focus (2005).

The lesson contained six stations. Each involved a different chemistry concept and utilized Minecraft's mechanics varyingly to gauge which implementations were effective. 


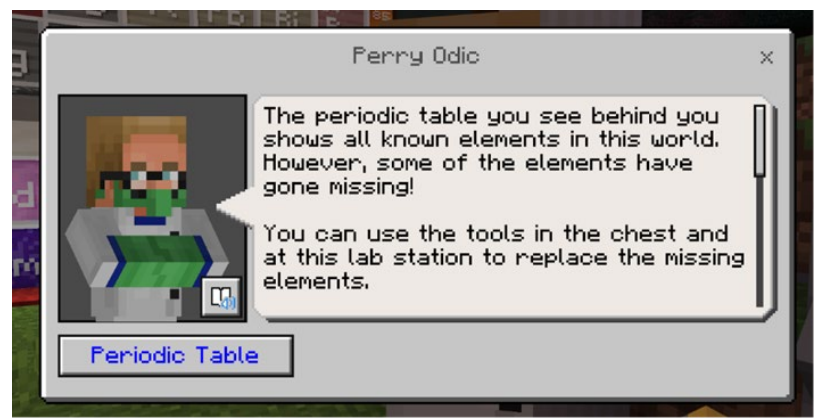

Figure 2: An NPC provides students information on the Figure periodic table in the red station.

Each station was color-coded for efficient tracking. To provide a control, certain stations emulated "drill and practice" education games, providing necessary information but leaving participants to decipher the specifics of the station's solution (Kiili, 2005). These were modeled after several PhET simulations (University of Colorado Boulder, 2021). Other stations heavily incorporated Minecraft's game design mechanics, having participants mine, craft, explore, and survive in the Minecraft Map to complete the objective (Duncan, 2011). However, all stations were partially experimental as the nature of the lesson meant they all incorporated Minecraft's mechanics by default. This was similar to the Minecraft lesson Circuit Madness, mentioned in Duncan's (2011) study. Circuit Madness contained several rooms, each implementing Minecraft's game design features for a non-Minecraft-related task (Duncan, 2011). However, the lesson in this study differed from Circuit Madness as it was intended to teach chemistry, not circuitry, and implemented Minecraft's feature of survival, which Circuit Madness did not (Duncan 2011). Circuit Madness also used a more linear model, having players progress through rooms in order, while this lesson was more openended (Duncan 2011). However, certain elements provided participants some direction.

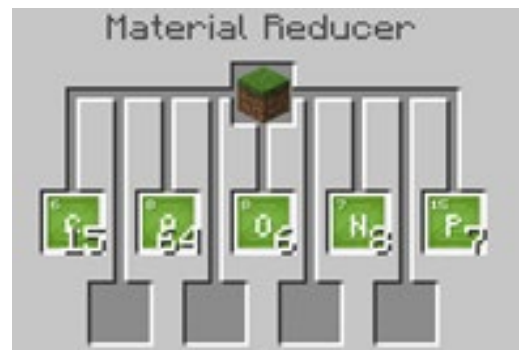

Figure 3: The Material Reducer in use, turning a Grass Block into several elements.

Each station had NPCs (Non-Playable Characters) with dialogue about each station (Figure 2). The lesson's NPCs recited information on the chemistry subject of the station as well as instructions on how to complete the station. Other Minecraft education maps incorporate NPCs, and they are currently the most efficient way to provide information in a Minecraft-based lesson (GoE-Craft, 2020). The elements allowed a connection between different phases of the game and integrated Minecraft's mechanics so each station could be viable but still experimental. For a more open-ended experience, participants were not required to complete each station.

Figure 4: The visualized periodic table in the red station, with missing elements to add.

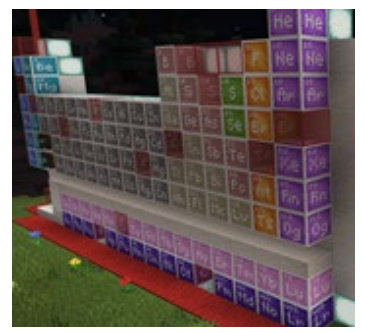




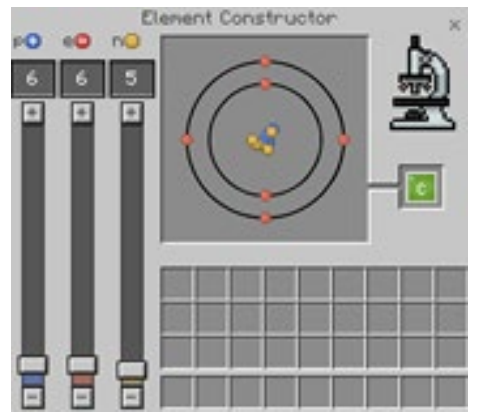

Figure 5: The Element Constructor in use.

\section{Individual stations:}

Red:

The red station introduced participants to the lesson and the periodic table. It was intended to teach everything in the world is made of elements. Participants were encouraged to explore the map and mine blocks, then use Minecraft Education Edition's material reducer, which turned "blocks", the objects that make up a Minecraft world, into their base elements (Mojang, 2016). Like in the base version of Minecraft, the "mining" mechanic of collecting blocks would have acted as a "first step" for many players during the lesson (Mojang, 2009). In this station, participants were asked to replace missing elements in a visual of the periodic table. The connection between the Minecraft mechanic of blocks and real-life elements may allow for deeper understanding. Furthermore, according to Watson et al.'s (2011) study, visuals of content engaged students more than a traditional lesson in history game-based learning, so this element was important to test in a Minecraft-based lesson. Additionally, participants could create elements to complete the red station in the orange station.

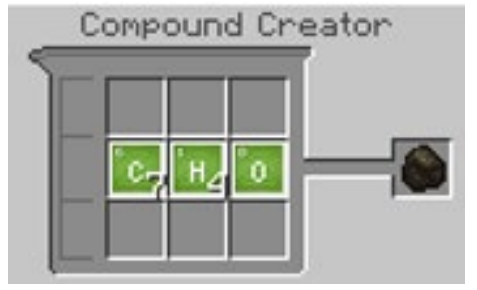

Figure 6: The Compound Creator in use to create charcoal, a useful item in Minecraft.

\section{Orange:}

The orange station taught participants how atoms are structured through Minecraft Education Edition's element constructor, and how to make elements using it. These elements could be used to complete the red station, furthering connections between topics, and offering the participant more options, which was intended to create a more open-ended lesson, similar to Civilization III, the open-ended game in Shree et al.'s (2008) study. This station involved the least exploration but integrated its specific mechanic heavily, as creating elements was its only activity.

\section{Yellow:}

The yellow station taught participants about chemical compounds, how they form, and how to create them using Minecraft Education Edition's compound creator. This station involved the most "drill and practice": similar to using a compound creating PhET simulation as a supplementary tool, students were provided information to legitimately solve the puzzle, but trial and error appeared more efficient (Kiili, 2005; University of Colorado Boulder, 2021). This was important to implement as Minecraft Education Edition does not provide information on how compounds form by default. 


\section{Green:}

The green station taught about chemical reactions and participants were required to use various lab equipment they had encountered to create a sparkler, an in-game item that provided light, which is important for survival and exploration in Minecraft (Mojang, 2009). It incorporated "drill and practice" using the same method as the yellow station, but involved more steps, and required participants to complete all steps to solve the station.

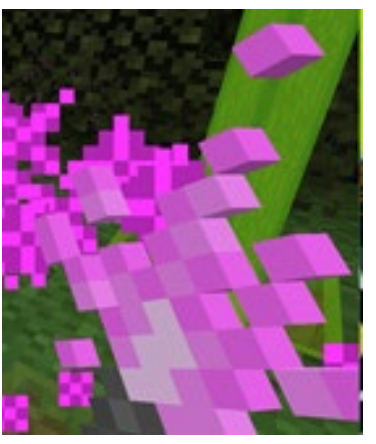

Figure 7: A lit sparkler in the green station

Blue:

The blue station involved elements, compounds, and reactions. This station incorporated the most exploration, as students were required to travel within a mountainous and ocean environment to receive all information needed to complete the station. This station contained many steps at different locations, providing multiple activities relating to the station's goal, which would increase flow according to Kiili (2005).

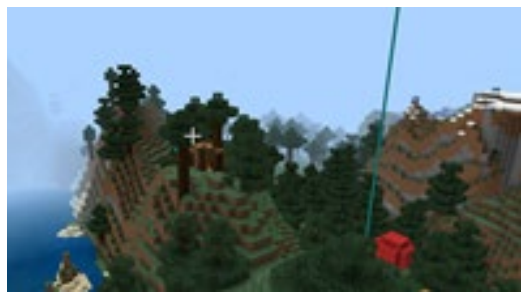

Figure 8: The area to be explored in the blue station.

\section{Purple:}

The purple station covered all topics in the station and was intended as the final station in the lesson. It had participants complete multiple steps and utilize all Minecraft Education Edition's lab equipment to finally create an item using the Lab Table, using the same principles as the blue station, minus the exploration.

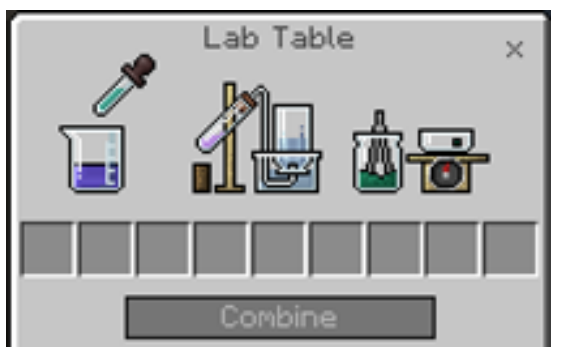

Figure 9: The lab table in the purple station. 


\section{Post-lesson Quiz:}

To evaluate the effectiveness of the lesson, a quiz was given to participants, providing quantitative results and emulating an assignment that would be given to students in class, similar to how Watson et al. (2011) collected assignments and tests of the students in their case study to observe how students. To judge how well each station taught the topic, questions linked to different stations (Figure 2). An "I don't know" response was available to see if the student was not able to retain information from the station linked to a question at all.

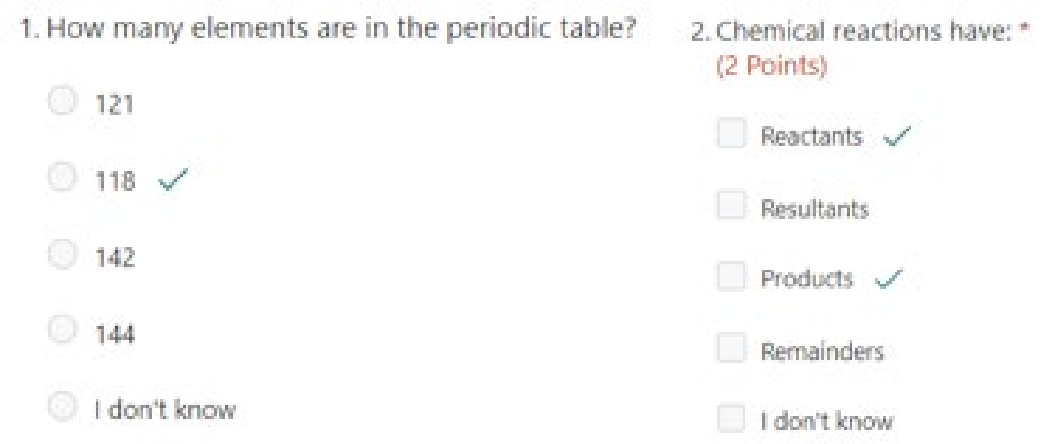

Figure 10: Questions 1 and 2 of the post-lesson quiz, which were linked to the red (periodic table) and green (chemical reactions) stations respectively.

\section{Reflection Survey:}

A reflection survey provided qualitative results and was created to emulate the written reflection students were given in Maguth et al.'s (2015) study, which allowed students to connect their understanding of a subject to learning. This was important to implement as "remote observation" did not allow for direct observation of participants, so their thoughts were necessary to consider. This survey contained questions regarding participants' prior chemistry knowledge to gauge how much they learned and Minecraft experience. Prior experience and familiarity with a game affect how students learn with it (Kiili, 2005). As Minecraft is a popular game, this likely affected students' interaction with the lesson (Hofer, 2015). Additionally, the reflection survey asked students to rate the stations based on effectiveness and engagement on a scale of 1-10, with 10 being the most effective/engaging, and discuss why these stations were effective or ineffective, so this information could be recorded as well. As each station utilized different mechanics, the final survey would allow the researcher to determine which ones were beneficial or not.

\section{Results and Analysis}

\begin{tabular}{|c|c|c|c|}
\hline Participant & Grade & High School Chemistry Level & Minecraft Experience \\
\hline 1 & 12 & Completed & Formerly played regularly \\
\hline 2 & 10 & Currently in chemistry class & Plays regularly \\
\hline 3 & 12 & Completed & Plays regularly \\
\hline 4 & 9 & No high school chemistry experience &
\end{tabular}

Figure 11: Description of each student who completed each part of the study. 
Out of the four students who completed the study, participants 1 and 3 had completed a high school chemistry class. Participant 2 was currently in a chemistry class, and participant 4 did not take high school chemistry. All participants were previously familiar with Minecraft.

No participant received a perfect score on the quiz, consistently getting questions linked to purposely ineffective control stations incorrect, showing the "drill and practice" principle in Kiili's study is ineffective compared to the more experimental stations. Most "I don't know" responses were from stations a participant did not complete.

Participant 1 received a score of 13 , which may have been due to prior chemistry experience, but this does not correspond with participant 3's score of 7. This was likely due to participant 1's effectiveness rating of 10 - they stated the NPCs in the stations they rated the most effective had enough information for the quiz, which was unrelated to game design elements in the lesson. Additionally, participant 1 only completed the Red station, further showing their success was not due to their in-game experience, contrasting Watson et al. (2011). and Maguth et al.'s (2015) studies, where the games led to a heightened learning experience. Participant 2 received a score of 10, with some incorrect answers on experimental stations using the game design element of exploration. This sentiment was shared with participant 3, who noted the lesson was difficult to navigate, indicating exploration may not mesh with learning. Exploration is a mechanic intertwined with a game being open-ended, but these results contrasted Shree et al.'s (2008) study where the open-ended game Civilization III was able to teach effectively. However, Civilization III allowed students to "live" through events they were required to learn about, a feature Minecraft does not share, which might explain why participants disliked the element of exploration (Shree et al., 2008).

\begin{tabular}{c|c|c|c}
\hline Participant & Quiz Score & Lesson Rating (Engagement) & Lesson Rating (Effectiveness) \\
\hline 1 & 13 & 4 & 10 \\
\hline 2 & 10 & 5 & 4 \\
\hline 3 & 7 & 5 & 4 \\
\hline 4 & 13 & 10 & 9 \\
\hline
\end{tabular}

Figure 12: Participants' quiz scores, and what they rated the lesson's fun/effectiveness.

Greatly contrasting other results, Participant 4 received 13 points without high school chemistry knowledge, showing learning effectively is possible with this integration of Minecraft's design elements and chemistry learning. One question they missed was related to the blue station, which they rated as "somewhat engaging" rather than the "very engaging" they rated all other stations, showing engagement Minecraft Education Edition provides can impact a student's learning, revealing a similar experience as students in Watson et al.'s (2011) study, where engagement the game provided affected the way students learned with it.

The effectiveness rating was split. Participants 1 and 4 rated the lesson 10 and 9, respectively. However, participants 2 and 3 both rated it as 4/10. Participant 1's rating may be explained by their previously mentioned statements, but participant 4's appears to be from their experience with the lesson's activities. This both contrasted the effectiveness of the games in Shree et al. (2008), Watson et al. (2011), and Maguth et al.'s (2015) studies and corresponded with the ineffectiveness of pre-existing education maps (GoE-Craft, 2020; Shapescape, 2020). However, as the results were split, Minecraft Education Edition's mechanics viability in creating an effective learning experience cannot be determined.

The engagement rating of the lesson was consistent, with participant 1 rating it a 4, and participants 2 and 3 rating it a 5. It could be said Minecraft Education Edition's game design elements within a chemistry lesson are not 
engaging - this may be due to a "chocolate-covered broccoli" situation, as discussed in Arena's study, which would indicate Minecraft's game design elements were mismatched when mixed with chemistry learning. However, participant 4 was an outlier in this category, rating the lesson a 10 for engagement, indicating engagement is possible within a Minecraft-based lesson, but participant 4 did not state why they rated the lesson highly.

Some participants discussed certain aspects of the lesson. Participant 1 stated "It was interesting to try and do the activities", indicating the lesson provided engagement but not enough for enjoyment. Participant 3 stated the effectiveness of the red station was due to it showing the periodic table, indicating the visual provided a better learning experience, similar to how a visual of the content enhanced learning experience in Watson et al.'s (2011) study. Participant 4 stated the many steps in the blue and purple stations made them more engaging. Blue's steps were largely due to its element of exploration, which contrasted with other participants' opinions on the mechanic. Participant 1 found the lesson "annoying to get around and hard to see where everything was" and did not learn well with the lack of direction. Participant 2 was also unable to work well with the lesson due to a lack of direction. These responses indicate a Minecraft-based lesson would need strict direction on objectives, but there are not enough responses to fully garner a conclusion. While these results provided information on Minecraft's mechanics, results were scarce and too mixed to determine which were truly effective.

Overall, the study's low sample size caused a lack of data, and collected data was incredibly varied. Although all participants were familiar with Minecraft, each interacted uniquely with the lesson. After analysis, Minecraft's game design elements may not mesh well with teaching chemistry to create an effective and engaging learning experience, as most participants were not engaged with the lesson, and 2 could not successfully learn within it. However, due to participant 4's experience being an outlier, results were heavily mixed, and as participant 4 did not have prior chemistry knowledge, successful learning is possible within a Minecraft-based lesson, but it is unclear how based on this study's results. The low sample size and mixed results make it difficult to determine whether Minecraft Education Edition's mechanics were able to create an engaging and viable lesson, but other factors may lead to a conclusion.

\section{Limitations, Implications \& Conclusion}

Due to COVID-19 and technology limitations, an in-person method was not feasible, and data that could have been analyzed was not gathered. Students could not be monitored during their progress through the lesson like in Watson et al. (2011) and Maguth et al.'s (2015) studies, limiting how their experiences with each part of the lesson were observed. Furthermore, these limitations permitted only a small portion of the school the study took place in to participate. Although the method used was theoretically effective as it was modeled after Maguth et al. (2015) and Watson et al.'s (2011) previously successful studies, sample size diminished its potential. Additionally, all participants were familiar with Minecraft, but in a real classroom environment, not all students would have played the game. In addition, as prior experience with a game affects how a student learns with it, this likely influenced the results (Kiili, 2005). In addition, most students had high school chemistry experience, which likely affected their results on the quiz. A direct-observation method of a class of pre-high school chemistry students would prevent these limitations in the future, providing a larger sample size and more data for a solid conclusion. Aditionally, it was not feasible within the length and sample size of the study to create a fully experimental lesson and test it alongside a control lesson. The inclusion of the intentionally frustrating stations may have lowered participants' overall perception of the lesson. In future research, control and experimental stations should be kept in separate lessons, or a purely experimental model should be tested.

Furthermore, more research should have been done on what game mechanics engage players and successfully induce student-centered learning. It was not feasible within the length or budget of this study to evaluate engaging game-based learning mechanics firsthand, and most information was taken from studies covering successful instances of game-based learning. Due to this, the lesson's experimental aspects were likely less effective. Future 
implementations of Minecraft game-based learning and future studies similar to this one should review various games to determine how their mechanics might be implemented within the lesson.

The results of this study imply successful learning is possible and Minecraft's mechanics may not mismatch with learning, as seen in participant 4's case. Therefore, this study provides an example of how Minecraft Education Edition can be implemented within a lesson, but as the lesson was highly seen as non-fun by other participants, it does not show how it should be. Moreover, the results show students had varied engagements with Minecraft Education Edition's features during the lesson. To provide an effective lesson for everyone, this variation of results should be considered in future implementations of Minecraft Education Edition and chemistry learning.

The answer to why the results were so varied may come from Minecraft's developers themselves, who state "We actually don't believe Minecraft is successful because all of it appeals to everyone. We think it's successful because it contains something for everyone" (Mojang, 2021). The initial research question overlooked Minecraft's complexity as a game, and while it did implement many aspects of Minecraft, the lesson created for the study does not appeal to most participants, and much of the lesson's nature may have skewed the results. As a very varied game with a wide range of appeal, it is reasonable to conclude not all of Minecraft's game mechanics appeal to all players. While survival and exploration, the two mechanics touched upon in this study, might be fun for some, they may be frustrating for others, and mechanics that were not focused on in this study may be incredibly fun to certain people. Additionally, Coleman and Money's (2019) study states “There remains a need to tie together more tightly the design of [games intended for education] with existing research on how students learn". From the received results and this information, Minecraft's game mechanics can provide engagement and an effective learning experience depending on how a student learns. However, the sample size was too small, and the results were too varied to garner a full conclusion. While the study considered how students learn, when tied with the study's heavily varied results and the fact Minecraft appeals differently to everyone, more data should be collected with a method addressing the previously mentioned limitations so it can be fully determined whether Minecraft Education Edition is able to provide an effective and engaging learning experience. This study provides a start, but future research must be done on how to create an implementation of Minecraft Education Edition's chemistry game design features that reach its potential for everyone so effective, student-centered, game-based learning for STEM is available.

\section{Acknowledgements}

I would like to thank Jill Berge for being an amazing teacher and mentor throughout AP Capstone and providing the resources I needed to write this paper, as well as Ronica Wilson and Melissa Mittan for requesting their students to participate in the study, the 4 participants who fully completed the study, allowing me to receive the data I needed, and my brother, who taught me about some of Minecraft's mechanics I was not fully familiar with. I would also like to express my gratitude towards Mojang for creating Minecraft and Minecraft Education Edition, the editors of the Minecraft wiki for providing incredibly comprehensive information on Minecraft Education Edition's items, and all the community members who created Minecraft modules for education, providing information on what a lesson could look like within Minecraft and a basis for this study.

\section{References}

Arena, D. (2015). Video games as tillers of soil. Theory Into Practice, 54(2), 94-100. https://doi.org/10.1080/00405841.2015.1010843

Burgos, D., Tattersall, C., \& Koper, R. (2007). Re-purposing existing generic games and simulations for elearning. Computers in Human Behavior. https://doi.org/10.1016/j.chb.2006.08.002

Clemenson, Gregory D, Henningfield, Caden M, \& Stark, Craig E L. (2019). Improving hippocampal memory through the experience of a rich Minecraft environment. Frontiers in Behavioral Neuroscience, 13, 57. 
Coleman, T. E., \& Money, A. G. (2020). Student-centred digital game-based learning: A conceptual framework and survey of the state of the art. Higher Education (00181560), 79(3), 415-457. https://doi.org/10.1007/s10734-019-00417-0

Duncan, S. C. (2011). Minecraft, beyond construction and survival. Well Played. https://se4n.org/papers/duncanminecraft.pdf

GoE-Craft. (2020). The human body [Video Game]. https://www.playthismap.com/products/goe-craft/the-humanbody

Gray, P., Ph.D. (2018, March 27). Benefits of play revealed in research on video gaming. Psychology Today. https://www.psychologytoday.com/us/blog/freedom-learn/201803/benefits-play-revealed-in-researchvideo-gaming

Hofer, C. C. (2015). Crafty chemistry: Science adds a surprising twist to the world of Minecraft. Science World (1987), 71(12), 12.

Kiili, K. (2005). Digital game-based learning: Towards an experiential gaming model. The Internet and Higher Education., 8(1), 13-24.

Maguth, B. M., List, J. S., \& Wunderle, M. (2015). Teaching social studies with video games. Social Studies, 106(1), 32-36. https://doi.org/10.1080/00377996.2014.961996

Mojang. (2016). Minecraft education edition [Video Game].

Mojang. (2009). Minecraft [Video Game].

Mojang. (2021, February 26). Redstone: Hoaw we make Minecraft - episode 7 [Video]. https://www.youtube.com/watch?v=DLSeGbecKMw

Nebel, S., Schneider, S., \& Rey, G. D. (2016). Mining learning and crafting scientific experiments: A literature review on the use of Minecraft in education and research. Journal of Educational Technology \& Society, 19(2), 355-366.

Shapescape. (2020). Astronaut training center [Video Game]. https://www.playthismap.com/products/shapescape/astronaut-training-center

Squire, K. D., DeVane, B., \& Durga, S. (2008). Designing centers of expertise for academic learning through video games. Theory Into Practice, 47. https://doi.org/10.1080/00405840802153973

University of Colorado Boulder, P. (2021). About PhET. PhET Interactive Simulations. https://phet.colorado.edu/en/about

Watson, W. R., Mong, C. J., \& Harris, C. A. (2011). A case study of the in-class use of a video game for teaching high school history. Computers \& Education, 56(2), 466-474. https://doi.org/10.1016/j.compedu.2010.09.007

Why you should be using video games in the classroom. (2014, July 14). Stemfuse. Retrieved September 4, 2020, from https://stemfuse.com/blog/why-you-should-be-using-video-games-classroom. 